\author{
Dries Testelmans \\ Karen Maes \\ Patrick Wouters \\ Scott K. Powers \\ Marc Decramer \\ Ghislaine Gayan-Ramirez
}

\section{Infusions of rocuronium and cisatracurium exert different effects on rat diaphragm function}

Received: 18 October 2006

Accepted: 12 February 2007

Published online: 15 March 2007

(C) Springer-Verlag 2007
D. Testelmans · K. Maes · M. Decramer .

G. Gayan-Ramirez ( $)$

Katholieke Universiteit Leuven, Respiratory

Muscle Research Unit, Laboratory of

Pneumology and Respiratory Division,

O\&N 1, bus 706, Herestraat 49,

3000 Leuven, Belgium

e-mail:

ghislaine.gayan-ramirez@med.kuleuven.be

Tel.: +32-16-330193

Fax: +32-16-347126

P. Wouters

Katholieke Universiteit Leuven,

Department of Anesthesiology,

UZ Gasthuisberg, Herestraat 49,

3000 Leuven, Belgium

S. K. Powers

University of Florida, Department of

Applied Physiology and Kinesiology,

Gainesville FL, USA

\begin{abstract}
Objective: Aminosteroidal and benzylisoquinoline neuromuscular blocking agents are used in the intensive care unit to facilitate mechanical ventilation. The use of these agents has been associated with development of critical illness myopathy; however, the relative frequency of myopathy development among agents is not known. The aim of our study was to compare the effects of $24 \mathrm{~h}$ infusion of rocuronium or cisatracurium on the diaphragm in mechanically ventilated rats. Design: Randomized, controlled experiment. Setting: Basic animal science laboratory. Subjects: Male Wistar rats, 14 weeks old. Interventions: Rats were divided into four groups to receive either saline, rocuronium (low dose) or cisatracurium (low or high dose). Measurements and results: After $24 \mathrm{~h}$, in vitro diaphragm tetanic force was decreased after rocuronium ( $-33 \%$ vs. saline), while the force was more preserved after cisatracurium, even in the high-dose
\end{abstract}

group. Cross-sectional areas of the different diaphragm and gastrocnemius fibers were unaltered. Diaphragmatic MURF-1 mRNA was increased after rocuronium $(+44 \%$ vs. saline), while unchanged in both cisatracurium groups. Calpain activity was increased after rocuronium $(+75 \%$ vs. saline) and unchanged in the cisatracurium groups. MURF-1 mRNA expression and calpain activity were negatively correlated with diaphragm force. Conclusions: Cisatracurium infusion during controlled mechanical ventilation exerted less detrimental effects on diaphragm function and proteolytic activity than infusion of rocuronium, even with the higher effective dose. These data suggest that increased calpain activity and increased activation of the ubiquitin proteasome system play a role in the different effects of these agents.

Keywords Mechanical ventilation . Neuromuscular blocking agent . Diaphragm

\section{Introduction}

Sustained administration of non-depolarizing neuromuscular blocking agents (NMBAs) can be used to facilitate mechanical ventilation (MV), assist in the treatment of increased intracranial pressure, or reduce oxygen consumption [1]. A large recent prospective cohort study showed that these agents are used in $13 \%$ of MV patients with a mean duration of 2 days [2]. In patients with acute respiratory distress syndrome the frequency $( \pm 25 \%)$ and duration of NMBA use is even higher [3]. Both aminosteroidal and benzylisoquinoline NMBAs are being used for this purpose, and clinical preference for a specific non-depolarizing NMBA is often based on the pharmacokinetic properties [4]. NMBA use is a known trigger for myopathy development which can lead to prolonged MV and intensive care unit (ICU) stay [1, 5]. After different case reports, this myopathy was originally attributed to the use of aminosteroidal NMBAs [3, 6]. However, benzylisoquinoline agents have also been associated with 
myopathy development $[7,8]$. Similar prevalence rates of persistent muscle weakness were shown in patients treated with vecuronium and atracurium (an aminosteroidal and a benzylisoquinoline agent respectively) [9], but in another prospective trial, no occurrence of myopathy was described in 153 patients receiving benzylisoquinoline agents [10].

Animal models of MV have shown that deleterious effects of controlled MV (CMV), including reduction in diaphragmatic force and fiber dimensions, developed after only 18-72 h of CMV [11, 12]. Additionally, we recently showed that rocuronium infusion during $24 \mathrm{~h}$ of CMV led to an additional decrease in diaphragm force and increased expression of diaphragmatic MURF-1 mRNA, an E3-ligase of the ubiquitin-proteasome pathway, suggesting enhanced muscle protein degradation after rocuronium [13]. Whether these effects result from the neuromuscular blocking process by itself and are induced by all non-depolarizing NMBAs, or from a pleiotropic effect of rocuronium, linked to its chemical structure, has not yet been clarified. Since aminosteroidal agents possess a steroid core and steroids are linked to myopathy development [14], the aminosteroidal structure might be responsible for the diaphragm dysfunction after rocuronium infusion. In this case, benzylisoquinoline NMBAs, with a similar neuromuscular blocking effect, would not cause these deleterious effects even when administered at a higher effective dose.

In the present study we compared the effects of two non-depolarizing NMBAs: rocuronium, an aminosteroidal NMBA and cisatracurium, a benzylisoquinoline derivative, on diaphragm function, histology and expression of proteolysis markers, to examine the extent to which these two NMBAs would affect diaphragm properties. In addition to measuring MURF-1 expression, calpain activity and the expression of its endogenous inhibitor, calpastatin, were examined.

Some results of this study have been previously reported in abstract form [15].

\section{Materials and Methods}

Experimental procedures, study design

The study was approved by the Animal Experiments Committee of the Medical Faculty of the Katholieke Universiteit, Leuven.

The study was performed on anesthetized adult male Wistar rats (14 weeks old) submitted to CMV for $24 \mathrm{~h}$ and receiving a continuous infusion of either $\mathrm{NaCl} 0.9 \%$ $(n=6)$, rocuronium in low dose $(n=6)$ or cisatracurium in low dose $(n=7)$ or high dose $(n=5)$.
The experimental setup was adapted from previous experiments [16]. Briefly, animals were anesthetized with sodium pentobarbital $\left(60 \mathrm{mg} \cdot \mathrm{kg}^{-1}\right.$ intraperitoneally) and tracheotomized. The right jugular vein and carotid artery were cannulated for infusion of sodium pentobarbital $\left(2 \mathrm{mg} .100 \mathrm{~g}^{-1} \cdot \mathrm{ml}^{-1}\right)$ and heparin $\left(2.8 \mathrm{U} \mathrm{ml}^{-1} \cdot \mathrm{h}^{-1}\right)$, respectively, and the right lateral tail vein for infusion of rocuronium, cisatracurium or $\mathrm{NaCl} 0.9 \%$. Blood pressure was checked every $2 \mathrm{~h}$ and blood gases every $12 \mathrm{~h}$. Around the left sciatic nerve, an electrode was implanted at the upper thigh level which, when stimulated, caused plantar flexion of the left foot [13]. Plantar flexion twitch, induced by supramaximal stimulation, was measured before infusion of rocuronium, cisatracurium or $\mathrm{NaCl} 0.9 \%$, after $1 \mathrm{~h}$, and then every $3 \mathrm{~h}$. Rocuronium and cisatracurium infusions were titrated to achieve a 50\% (rocuronium and low-dose cisatracurium) or $90 \%$ (high-dose cisatracurium) reduction in twitch.

Electromyographic diaphragmatic activity was measured, as previously described, in three animals of each group [13]. These measurements confirmed the absence of diaphragmatic electrical activity during CMV in all groups.

During the $24 \mathrm{~h}$, continuing care to the animals was performed including expressing the bladder and lubricating the eyes. Animals breathed humidified air $\left(37^{\circ} \mathrm{C}\right)$ enriched with $\mathrm{O}_{2}$. MV occurred in volume-control mode (tidal volume: $0.6 \mathrm{ml} 100 \mathrm{~g}^{-1}$ body weight, respiratory rate: $60 \mathrm{~min}^{-1}$ ).

After $24 \mathrm{~h}$, costal diaphragm segments were removed for measurement of in vitro contractile properties as previously described [17]. Briefly, two diaphragm bundles per animal were suspended in a tissue bath $\left(37^{\circ} \mathrm{C}\right)$ containing Krebs solution continuously aerated with $95 \% \mathrm{O}_{2}$ and $5 \% \mathrm{CO}_{2}$. Optimal length for peak twitch force was verified, and after a 15-min thermoequilibration period the force-frequency relationship was established by direct bundle stimulation at the following frequencies: $1,25,50$, 80,120 , and $160 \mathrm{~Hz}(250 \mathrm{~ms}$ train duration, $0.2 \mathrm{~ms}$ pulse duration) and fatigue properties were measured (330-ms stimulations at $25 \mathrm{~Hz}$ every $3 \mathrm{~s}$ for $5 \mathrm{~min}$ ). Cross-sectional area (CSA) was obtained by dividing bundle weight by muscle specific density and optimal length. Forces were expressed per unit CSA.

In addition, weights of the diaphragm, gastrocnemius and soleus muscle were measured. One part of the costal diaphragm was frozen in liquid nitrogen for further examination as described subsequently. The other part of the costal diaphragm and the gastrocnemius muscle were fold, cut transversely, and frozen in isopentane cooled with liquid nitrogen to subsequently perform histological and morphological analysis. Animals were killed by injecting a sodium pentobarbital bolus into the heart. 
Histology and histochemistry

Sections of diaphragm and gastrocnemius were cut at $10 \mu \mathrm{m}$ thickness with a cryostat maintained at $-20^{\circ} \mathrm{C}$. Sections were stained with hematoxylin and eosin (H\&E) and with myofibrillar adenosine triphosphatase (ATPase) after acid preincubation at $\mathrm{pH} 4.5$ and 4.3. CSA and proportion of slow-twitch type I, fast-twitch type IIa or IIx/b fibers were determined from the pixel number within the outlined borders using a Leitz Laborlux X. Microscope (Wetzlar, Germany) at $\times 20$ magnification, connected to a computerized image system (Quantimet 500, Leica, Cambridge, UK). Around 150 fibers were used to calculate CSA of all fiber types and proportion.

\section{RNA extraction and RT-PCR}

Total diaphragm RNA was extracted using TRIzol. After reverse transcription, semi-quantitative PCR was performed with MAFbx and MURF-1 primers, with $18 \mathrm{~S}$ ribosomal RNA as internal standard. The $18 \mathrm{~S}$ primers were mixed with competimers at an optimized ratio of 1:9. Amplification products were analyzed by electrophoresis stained with Vistra Green and quantified by fluorescence imaging (Photo-print, Vilber Lourmat, France). Intensities of the amplified fragments were normalized to the corresponding $18 \mathrm{~S}$ amplification signals.

\section{Calpain activity and calpastatin expression}

To assess diaphragm calpain activity, calpain-specific cleavage products of $\alpha \mathrm{II}$-spectrin [18] were analyzed with western blotting since activity assays are only reliable with large tissue samples [19]. After diaphragm homogenization in $\mathrm{KPO}_{4}$ buffer, equal amounts of protein were separated by SDS-polyacrylamide gel electrophoresis and transferred by semidry blotting. Blots were exposed to a monoclonal primary antibody to $\alpha$ II-spectrin (Biomol, PA). After washing, a HRP-conjugated anti-mouse secondary antibody was applied (Dako, Denmark). Bands at $260 \mathrm{kDa}$ represent intact $\alpha \mathrm{II}-$ spectrin and bands at 150 and $145 \mathrm{kDa}$ represent calpain-specific cleavage products of $\alpha$ II-spectrin [20]. Cleaved bands were expressed as percentage of intact bands and normalized as a percentage of the normal saline group. Diaphragmatic calpastatin expression was measured using polyclonal anti-calpastatin (Sigma) and a HRP-conjugated anti-rabbit IgG (Dako) as secondary antibody.

\section{Statistical analysis}

Statistical analysis was performed with the SAS statistical package (SAS Institute Cary, NC, USA). Data were tested for normality and equal variance. Comparisons among the four groups were performed using ANOVA with post hoc Newman-Keuls multiple comparison test. Correlations were assessed with the Pearson correlation coefficient. Data are presented as means $\pm \mathrm{SE}$, unless otherwise specified.

\section{Results}

Blood gases, arterial blood pressure and infusion doses

Blood gas/pH homeostasis was similar in the different groups and was kept within the normal range throughout the experiment. Mean arterial blood pressure was not different between the four groups (Table 1). The dose of sodium pentobarbital was similar in all groups (1.44 mg. $100 \mathrm{~g}^{-1} \cdot \mathrm{h}^{-1}$, pooled values). Mean rocuronium dose was $6.02 \mathrm{mg} \cdot \mathrm{kg}^{-1} \cdot \mathrm{h}^{-1}$. Mean cisatracurium doses were 2.67 and $3.51 \mathrm{mg} \cdot \mathrm{kg}^{-1} \cdot \mathrm{h}^{-1}$ in the low-dose cisatracurium and high- dose cisatracurium groups, respectively $(p<0.01)$.

Twitch of the gastrocnemius complex

Twitches decreased immediately to $50 \%$ of the initial value in the rocuronium and low-dose cisatracurium groups and
Table 1 Blood gas data, arterial blood pressure at dissection time and cross-sectional area (CSA) of diaphragm fiber types I, IIa and IIx/b in the normal saline, rocuronium, low-dose cisatracurium and high-dose cisatracurium groups. Values are mean \pm SD expressed in torr for blood gases and arterial blood pressure and in $\mu \mathrm{m}^{2}$ for CSA

\begin{tabular}{lcccc}
\hline & Normal saline & Rocuronium & $\begin{array}{l}\text { Low-dose } \\
\text { cisatracurium }\end{array}$ & $\begin{array}{c}\text { High-dose } \\
\text { cisatracurium }\end{array}$ \\
\hline $\mathrm{Blood}$ & & & & \\
$\mathrm{PaO}_{2}$ & $126 \pm 60$ & $122 \pm 17$ & $103 \pm 20$ & $104 \pm 23$ \\
$\mathrm{PaCO}_{2}$ & $41 \pm 3$ & $36 \pm 5$ & $40 \pm 10$ & $36 \pm 7$ \\
$\mathrm{pH}$ & $7.42 \pm 0.05$ & $7.47 \pm 07$ & $7.42 \pm 0.04$ & $7.42 \pm 0.08$ \\
Arterial blood pressure & $90.83 \pm 11.03$ & $103.2 \pm 6.08$ & $101.00 \pm 8.47$ & $99.00 \pm 13.19$ \\
Fiber type CSA & & & & \\
$\quad$ I & $753 \pm 105$ & $745 \pm 59$ & $742 \pm 82$ & $773 \pm 84$ \\
$\quad$ IIa & $876 \pm 101$ & $872 \pm 73$ & $858 \pm 112$ & $893 \pm 110$ \\
$\quad$ IIx/b & $1853 \pm 156$ & $1697 \pm 109$ & $1857 \pm 129$ & $1817 \pm 327$ \\
\hline
\end{tabular}




\section{*}

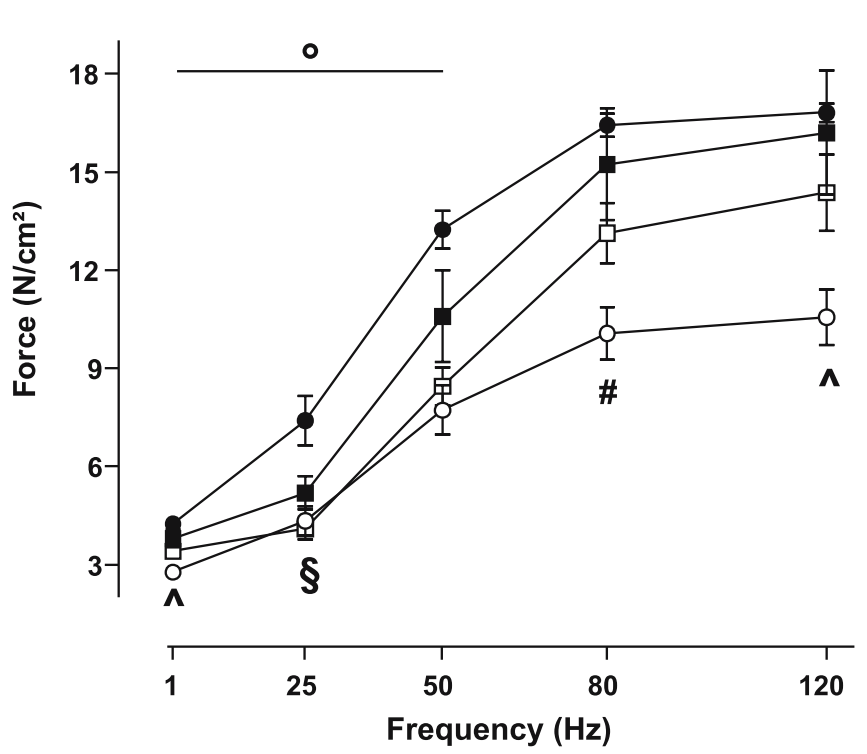

Fig. 1 In vitro force-frequency relationship of the diaphragm expressed in absolute values in the normal saline (closed circles), rocuronium (open circles), low-dose cisatracurium (closed squares) and high-dose cisatracurium (open squares) groups. Values are means and SE. $* p<0.01$ rocuronium vs. normal saline, $\# p<0.01$ rocuronium vs. low-dose cisatracurium, $\hat{p} p<0.05$ rocuronium vs. low-dose cisatracurium and high-dose cisatracurium, ${ }^{\circ} p<0.05$ high-dose cisatracurium vs. normal saline, $\$ p<0.01$ low-dose cisatracurium vs. normal saline

to $10 \%$ of the initial value in the high-dose cisatracurium group and were kept around this level during the entire experiment. Twitches in rocuronium, low-dose cisatracurium and high-dose cisatracurium groups were different at all time points in comparison with the normal saline group. There was no difference in twitch between the rocuronium and low-dose cisatracurium groups.

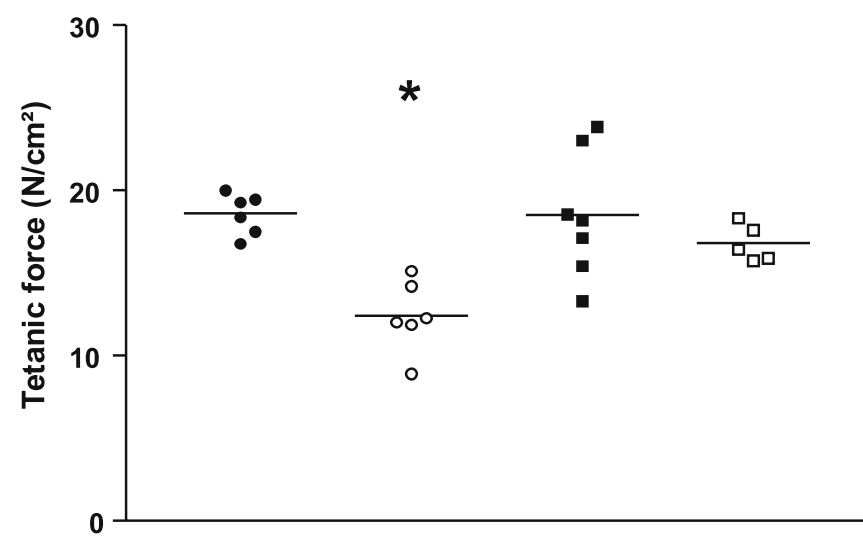

Fig. 2 In vitro diaphragm tetanic force in the normal saline, rocuronium, low-dose cisatracurium and high-dose cisatracurium groups (same symbols as in Fig. 1). Each point represents an individual animal. The horizontal line represents the mean value of the group. $* p<0.01$ vs. all other groups
Diaphragm contractile properties

In the rocuronium group, in vitro diaphragm force was reduced in comparison with the normal saline group at all stimulation frequencies (Fig. 1), including tetanic tension (Fig. 2). Compared with both cisatracurium groups, diaphragm force in the rocuronium group was lower at $1 \mathrm{~Hz}$ and at the high stimulation frequencies (Fig. 1). In comparison with the normal saline group, force was reduced in the low-dose cisatracurium group at $25 \mathrm{~Hz}$ and in the high-dose cisatracurium group at the low stimulation frequencies. Forces in low-dose cisatracurium and highdose cisatracurium groups were not different. The fatigue properties of the diaphragm were similar in all the groups.

\section{Histology of diaphragm and gastrocnemius}

H\&E staining revealed no pathologic changes in the diaphragm and the gastrocnemius muscle. The proportion of diaphragm and gastrocnemius fibers remained unchanged between the groups. There were no significant differences in CSA of the different diaphragm and gastrocnemius fiber types. However, CSA of type IIx/b fibers in the diaphragm was non-significantly decreased in the rocuronium group $(-9 \%)$ in comparison with the normal saline group ( $p>0.05$ vs. all groups) (Table 1$)$.
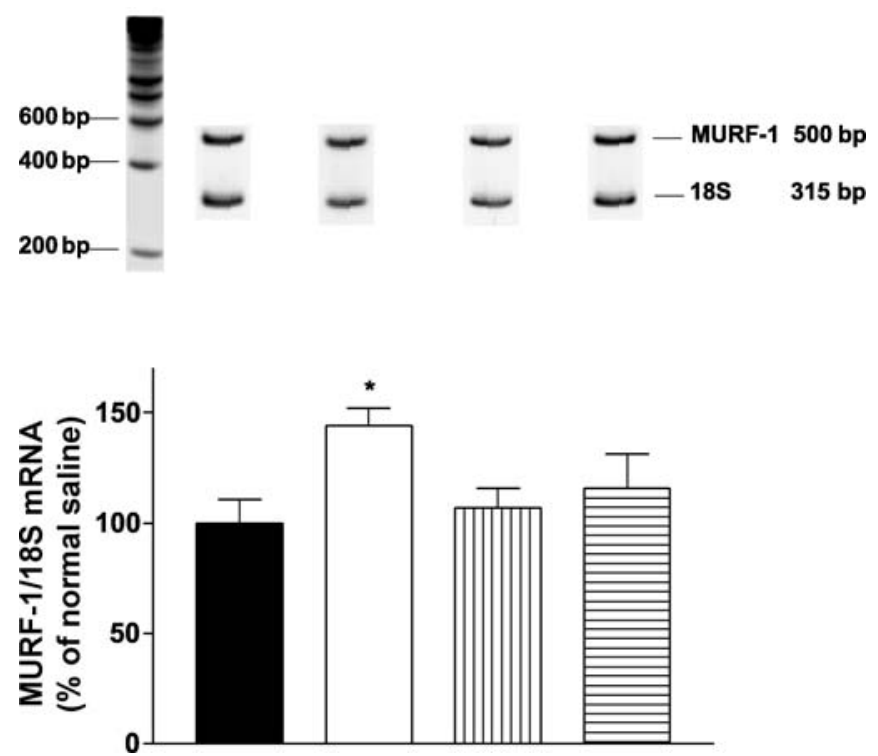

Fig. 3 Diaphragm MURF-1/18S mRNA expression in the normal saline (solid bar), rocuronium (open bar), low-dose cisatracurium (vertically hatched bar) and high-dose cisatracurium (horizontally hatched bar) groups. The left lane is the DNA ladder; the bottom bands (315 bp) represent the $18 \mathrm{~S}$ signal, the top bands $(500 \mathrm{bp})$, the MURF-1 signal of a representative animal in each group. Values are means and SE, expressed as percentage of the normal saline group. $* p<0.05$ vs. normal saline and low-dose cisatracurium 


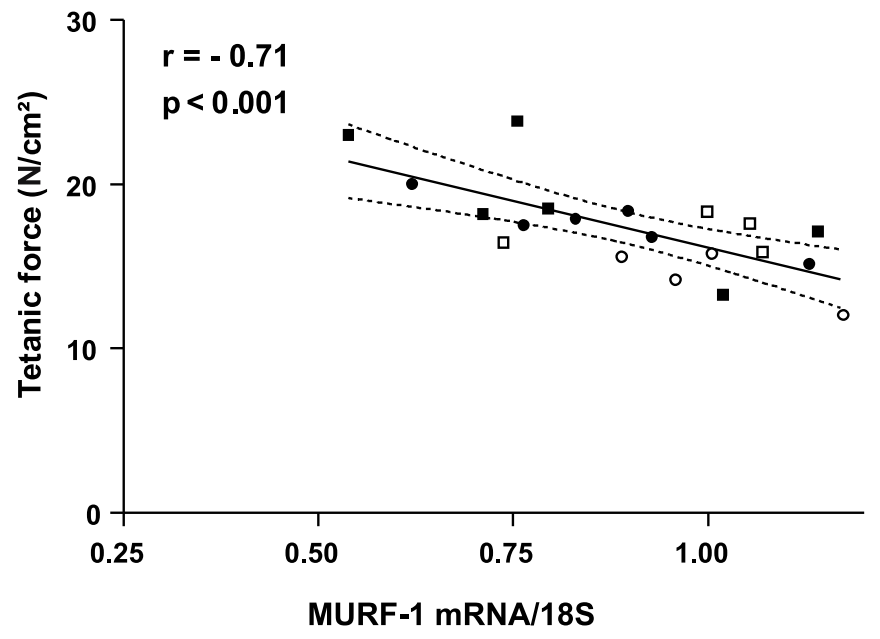

Fig. 4 Correlation between MURF-1 mRNA levels in the diaphragm and diaphragm tetanic force in normal saline (closed circles), rocuronium (open circles), low-dose cisatracurium (closed squares) and high-dose cisatracurium (open squares) groups. The solid line and dotted lines represent the regression line and 95\% confidence intervals, respectively

Expression of diaphragm MURF-1 and MAFbx/atrogin-1 mRNA

Expression of MAFbx mRNA was not different between the groups. MURF-1 mRNA expression in the diaphragm was increased by $44 \%(p<0.05$ vs. normal saline and
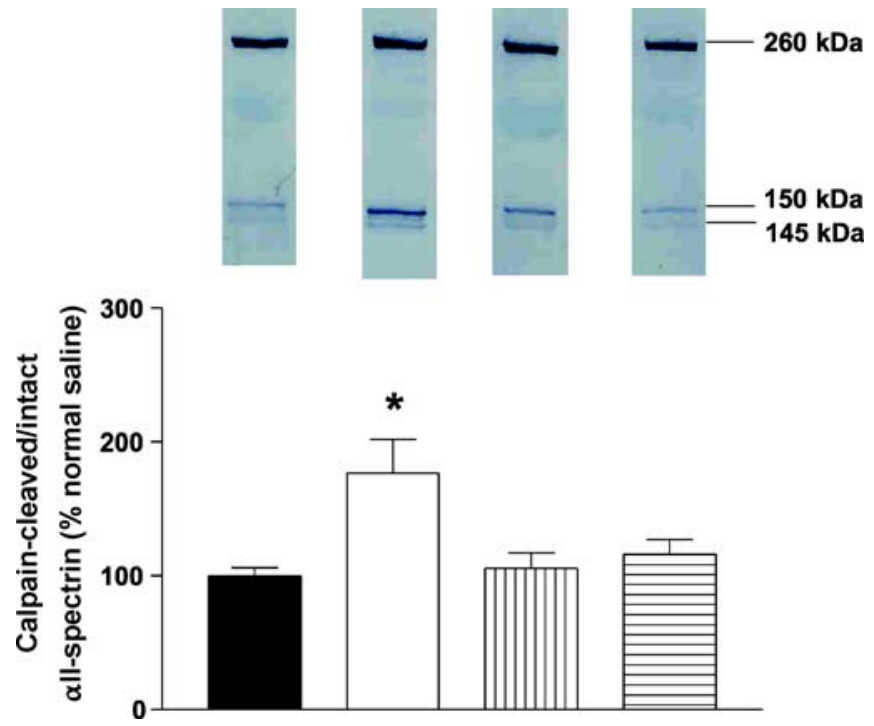

Fig. 5 Ratio of calpain-cleaved to total $\alpha$ II-spectrin present in the diaphragm of animals in the normal saline, rocuronium, low-dose cisatracurium and high-dose cisatracurium groups. Bars as in Fig. 3 . Bands at $260 \mathrm{kDa}$ represent intact $\alpha \mathrm{II}-$ spectrin, and bands at 150 and $145 \mathrm{kDa}$ represent calpain-cleaved $\alpha$ II-spectrin. Values (means and SE) are expressed as a percentage of the normal saline group. $* p<0.05$ vs. all other groups low-dose cisatracurium groups) in the rocuronium group while it remained unchanged in both cisatracurium groups (Fig. 3). There was an inverse correlation between diaphragmatic MURF-1 mRNA expression and diaphragm tetanic force $(r=-0.71, p=0.0005)$ (Fig. 4). Similar correlations were present for the other stimulation frequencies $(-0.71<r<-0.42, p<0.05)$ (data not shown).

Calpain activity in the diaphragm

The ratio of calpain-cleaved $\alpha$ II-spectrin to total $\alpha$ IIspectrin, a reliable marker of calpain activity [20], was higher in the diaphragm of the rocuronium group than in all other groups $(p<0.05)$ while unchanged in both cisatracurium groups (Fig. 5). An inverse correlation was found between the diaphragmatic tetanic force and the ratio of cleaved to total $\alpha$ II-spectrin in the diaphragm $(r=-0.49, p<0.05)$. Diaphragm levels of the endogenous calpain inhibitor calpastatin were similar among the four groups (51379 $\pm 3491 \mathrm{AU}$, pooled values).

\section{Discussion}

Overview of the principal findings

This study shows for the first time a direct comparison of the effects of two NMBAs with different chemical core structure on the diaphragm. The data show that continuous infusion of an aminosteroidal neuromuscular blocking agent (i.e. rocuronium) during the course of $24 \mathrm{~h}$ of CMV in rats is more deleterious for diaphragm force than continuous infusion of a similar effective dose of a benzylisoquinoline agent (i.e. cisatracurium). Even when the dose of cisatracurium is increased, the effect on diaphragm force is less detrimental than that of rocuronium at lower effective dose. Moreover, rocuronium increased the expression of MURF-1 mRNA and the calpain activity in the diaphragm, while there were no significant changes observed in the diaphragm of the rats treated with cisatracurium. These data suggest that the decreased diaphragm force after rocuronium infusion is the consequence of a direct effect of rocuronium on the muscle caused by an increased myofilament cleavage and proteolysis. It appears from our data that cisatracurium infusion during MV in rats is less harmful for the diaphragm than rocuronium infusion.

\section{Experimental model and clinical relevance}

The same animal model as in our previous study was used [13]. Electromyographic measurements confirmed that diaphragm activity was silenced in the different groups. The effective doses of rocuronium and cisatracurium used in this study are comparable with those 
used by clinicians in the ICU (one to three twitches with train-of-four testing) [3]. We chose rocuronium because it is the latest available aminosteroidal agent which has become popular for providing excellent intubating conditions in ICU patients [21]. Cisatracurium was chosen as the representative benzylisoquinoline agent because it has a similar duration of action as rocuronium and is also widely used in the ICU [22].

Guideline-based indications for the sustained use of NMBAs in the ICU are facilitation of MV, treatment of intracranial pressure and enhancement of oxygenation [1]. A recent study by Arroliga et al. showed that these agents were used in about $13 \%$ of mechanically ventilated patients with a mean duration of 2 days [2], and Prielipp et al. described that up to $10 \%$ of ICU patients receive continuous administration of NMBAs for at least $24 \mathrm{~h}$ [23]. Moreover, NMBAs are used in about $25 \%$ of mechanically ventilated patients with ARDS, especially in the early phase [24]. Since diaphragm measurements were performed after $24 \mathrm{~h}$ of MV and NMBA infusion, we cannot rule out the possibility that longer duration of cisatracurium infusion would exert greater effects on the diaphragm, but in any case the effects of rocuronium seem to be more marked for the same infusion duration. Whether the detrimental effects of rocuronium are reversible or not was not the subject of this study and cannot be concluded from these data.

\section{NMBAs and muscle}

Administration of NMBAs can lead to two patterns of severe muscle weakness: prolonged paralysis, characterized by an increased recovery time due to accumulation of NMBA or metabolites, and critical illness myopathy (CIM), a disorder in which skeletal muscle becomes electrically inexcitable even long after the NMBA and its metabolites are eliminated $[1,25]$. It was shown that CIM developed more frequently in patients who received NMBAs and that CIM significantly increased the duration of MV [24]. The relative frequency of CIM between different NMBAs has not yet been clarified. Aminosteroidal and benzylisoquinoline agents have both been associated with the development of CIM; in clinical studies, however, confounding factors are often present, such as co-administration of corticosteroids or antibiotics [26]. In our study, diaphragm force was significantly and severely reduced after $24 \mathrm{~h}$ infusion of rocuronium in a dose which causes a 50\% twitch reduction of a peripheral muscle, while this reduction in diaphragm force was less (lower stimulation frequencies) or not present (higher stimulation frequencies) after infusion of a functionally similar or higher dose of cisatracurium.
These findings point out that the effect of rocuronium on the diaphragm is direct and not related to a subsequent decrease in diaphragm activity. Moreover, EMG measurements showed absence of diaphragmatic activity in all groups.

Peripheral muscles of patients with CIM show presence of atrophy, especially of fast-twitch fibers [27]. In our study, there was no significant difference in CSA of the different fiber types among the groups. However, in accordance with our previous study [13], a slightly decreased CSA of type IIx/b fibers only was shown in the diaphragm of the rocuronium group compared with the normal saline group. The fact that NMBA infusion had no significant effect on diaphragm fiber CSA might be explained by the relatively short study duration and the fact that $24 \mathrm{~h}$ of CMV alone already induced atrophy of type I and IIx/b fibers [28].

\section{Muscle proteolysis}

Studies regarding skeletal muscle wasting in conditions of disuse and disease have shown that three proteolytic systems can induce muscle atrophy: the calcium-dependent calpain system, the lysosomal protein system and the ubiquitin-proteasome system. Diaphragm unloading by CMV activates all these proteolytic systems [29-32]. Also, activation of the calpain and the ubiquitin-proteasome system has been shown in CIM [33, 34].

MAFbx and MURF-1, two E3-ligases of the ubiquitin-proteasome system, have been identified as key substrate-specific enzymes in the ubiquitination process [35]. Regulation of these genes is used as a marker of muscle atrophy in different conditions of disuse and starvation [36]. In our study MURF-1 mRNA was upregulated in the rocuronium group, but unaltered in both cisatracurium groups. MAFbx was not upregulated by infusion of rocuronium or cisatracurium. The selective upregulation of MURF-1 mRNA in animals treated with rocuronium suggests activation of $\mathrm{NF} \kappa \mathrm{B}$, since it was previously shown that only MURF-1, and not MAFbx, was upregulated in muscle of transgenic mice overexpressing active IкB kinase, which allows activation of $\mathrm{NFKB}$ [37].

Since the ubiquitin-proteasome system is incapable of degrading intact myofibrillar proteins, intact myofibrils must first be cleaved by another proteolytic mechanism to become a substrate for the proteasome system [32]. Hereby, the proteasome and the calpain system seem to be regulated in parallel [38]. In agreement, an increased diaphragmatic calpain activity was found in the rocuronium group together with an increased MURF-1 expression. This increased calpain activity may lead to cleavage of 
intact myofibrils which can be further degraded by the ubiquitin-proteasome system [39].

Normally, the calpain activity in muscle fibers is regulated by interaction of the endogenous calpain inhibitor calpastatin and by free cytosolic calcium [40]. Since calpastatin protein levels did not differ among the four groups in our experiment, the increased calpain activity is probably caused by disturbances in calcium homeostasis of the muscle cells. Indirect evidence shows that intracellular calcium levels are increased in the diaphragm after CMV [29]. Moreover, since corticosterone, the principal glucocorticoid in rats, increases calcium uptake in muscle cells and aminosteroidal compounds are similar to corticosteroids with regard to molecular structure, rocuronium infusion could possibly be responsible for an increased intramuscular calcium level $[22,41]$.

The fact that diaphragmatic MURF-1 and the calpain activity are not upregulated in the cisatracurium groups underlines the importance of these markers in the pathophysiology of the diaphragm dysfunction induced by rocuronium.

\section{Conclusions}

In rats, $24 \mathrm{~h}$ infusion of cisatracurium, a benzylisoquinoline NMBA, exerted less detrimental effects on diaphragm function and proteolytic activity than rocuronium, an aminosteroidal agent. The use of prolonged infusions of rocuronium has been questioned recently because of the potential drug accumulation in patients with renal and/or hepatic failure [4]. Our data may provide another argument against this practice. Whether the effects of benzylisoquinoline and aminosteroidal drugs on diaphragmatic function and proteolytic activity also differ after short-term use in humans seems an important question that needs to be addressed in future studies.

Acknowledgements. The authors sincerely thank Mrs. Petra Weckx for cutting muscle samples and staining the histological sections. We also thank Prof. E. Verbeken for the evaluation of the H\&E-stained sections. This study was supported by FWOVlaanderen \#G.0389.03, KUL Research Foundation OT/02/44 and AstraZeneca Pharmaceuticals. Dries Testelmans is an aspirant of the "Fonds voor Wetenschappelijk Onderzoek - Vlaanderen"

\section{References}

1. Murray MJ, Cowen J, DeBlock H, Erstad B, Gray AW Jr, Tescher AN, McGee WT, Prielipp RC, Susla G, Jacobi J, Nasraway SA Jr, Lumb PD (2002) Clinical practice guidelines for sustained neuromuscular blockade in the adult critically ill patient. Crit Care Med 30:142-156

2. Arroliga A, Frutos-Vivar F, Hall J, Esteban A, Apezteguia C, Soto L, Anzueto A (2005) Use of sedatives and neuromuscular blockers in a cohort of patients receiving mechanical ventilation. Chest 128:496-506

3. Sessler CN (2005) Sedation, analgesia, and neuromuscular blockade for high-frequency oscillatory ventilation. Crit Care Med 33:S209-S216

4. Moore EW, Hunter JM (2001) The new neuromuscular blocking agents: do they offer any advantages? Br J Anaesth 87:912-925

5. De Jonghe B, Bastuji-Garin S, Sharshar T, Outin H, Brochard L (2004) Does ICU-acquired paresis lengthen weaning from mechanical ventilation? Intensive Care Med 30:1117-1121

6. Olivieri L, Plourde G (2005) Prolonged (more than ten hours) neuromuscular blockade after cardiac surgery: report of two cases. Can J Anaesth 52:88-93

7. Davis NA, Rodgers JE, Gonzalez ER, Fowler AA III (1998) Prolonged weakness after cisatracurium infusion: a case report. Crit Care Med 26:1290-1292
8. Tousignant CP, Bevan DR, Eisen AA, Fenwick JC, Tweedale MG (1995) Acute quadriparesis in an asthmatic treated with atracurium. Can J Anaesth 42:224-227

9. Leatherman JW, Fluegel WL, David WS, Davies SF, Iber C (1996) Muscle weakness in mechanically ventilated patients with severe asthma. Am J Respir Crit Care Med 153:1686-1690

10. Gehr LC, Sessler CN (2001) Neuromuscular blockade in the intensive care unit. Semin Respir Crit Care Med 22:175-188

11. Gayan-Ramirez G, Decramer M (2002) Effects of mechanical ventilation on diaphragm function and biology. Eur Respir J 20:1579-1586

12. Jaber S, Sebbane M, Koechlin C, Hayot M, Capdevila X, Eledjam JJ, Prefaut C, Ramonatxo M, Matecki S (2005) Effects of short vs. prolonged mechanical ventilation on antioxidant systems in piglet diaphragm. Intensive Care Med 31:1427-1433

13. Testelmans D, Maes K, Wouters P, Gosselin N, Deruisseau K, Powers S, Sciot R, Decramer M, GayanRamirez G (2006) Rocuronium exacerbates mechanical ventilationinduced diaphragm dysfunction in rats. Crit Care Med 34:3018-3023

14. Laghi F, Tobin MJ (2003) Disorders of the respiratory muscles. Am J Respir Crit Care Med 168:10-48
15. Testelmans D, Maes K, Wouters $P$, Decramer M, Gayan-Ramirez G (2006) Different effects of two families of neuromuscular blocking agents on rat diaphragm function during controlled mechanical ventilation. Proc Am Thoracic Soc [Abstract] 3:A137

16. Gayan-Ramirez G, De Paepe K, Cadot P, Decramer M (2003) Detrimental effects of short-term mechanical ventilation on diaphragm function and IGF-I mRNA in rats. Intensive Care Med 29:825-833

17. Dekhuijzen PN, Gayan-Ramirez G, de Bock V, Dom R, Decramer M (1993) Triamcinolone and prednisolone affect contractile properties and histopathology of rat diaphragm differently. J Clin Invest 92:1534-1542

18. Takamure M, Murata KY, Tamada Y, Azuma M, Ueno S (2005) Calpaindependent alpha-fodrin cleavage at the sarcolemma in muscle diseases. Muscle Nerve 32:303-309

19. Kent MP, Veiseth E, Therkildsen M, Koohmaraie M (2005) An assessment of extraction and assay techniques for quantification of calpain and calpastatin from small tissue samples. J Anim Sci 83:2182-2188

20. Czogalla A, Sikorski AF (2005) Spectrin and calpain: a 'target' and a 'sniper' in the pathology of neuronal cells. Cell Mol Life Sci 62:1913-1924 
21. Booij LH (2001) Is succinylcholine appropriate or obsolete in the intensive care unit? Crit Care 5:245-246

22. Whetstone Foster JG, Clark AP (2006) Functional recovery after neuromuscular blockade in mechanically ventilated critically ill patients. Heart Lung 35:178-189

23. Prielipp RC, Coursin DB, Wood KE, Murray MJ (1995) Complications associated with sedative and neuromuscular blocking drugs in critically ill patients. Crit Care Clin 11:983-1003

24. De Jonghe B, Sharshar T, Lefaucheur JP, Authier FJ, Durand-Zaleski I, Boussarsar M, Cerf C, Renaud E, Mesrati F, Carlet J, Raphael JC, Outin H Bastuji-Garin S (2002) Paresis acquired in the intensive care unit: a prospective multicenter study. JAMA 288:2859-2867

25. Filatov GN, Rich MM (2004) Hyperpolarized shifts in the voltage dependence of fast inactivation of Nav1.4 and Nav1.5 in a rat model of critical illness myopathy. J Physiol 559:813-820

26. Murray MJ, Brull SJ, Bolton CF (2006) Brief review: Nondepolarizing neuromuscular blocking drugs and critical illness myopathy. Can J Anaesth 53:1148-1156

27. Gutmann L, Blumenthal D, Gutmann L, Schochet SS (1996) Acute type II myofiber atrophy in critical illness. Neurology 46:819-821

28. Gayan-Ramirez G, Testelmans D, Maes K, Racz GZ, Cadot P, Zador E, Wuytack F, Decramer M (2005) Intermittent spontaneous breathing protects the rat diaphragm from mechanical ventilation effects. Crit Care Med 33:2804-2809
29. DeRuisseau KC, Shanely RA, Akunuri N, Hamilton MT, Van Gammeren D, Zergeroglu AM, McKenzie M, Powers SK (2005) Diaphragm unloading via controlled mechanical ventilation alters the gene expression profile. Am J Respir Crit Care Med 172:1267-1275

30. Shanely RA, Zergeroglu MA, Lennon SL, Sugiura T, Yimlamai T, Enns D, Belcastro A, Powers SK (2002) Mechanical ventilation-induced diaphragmatic atrophy is associated with oxidative injury and increased proteolytic activity. Am J Respir Crit Care Med 166:1369-1374

31. DeRuisseau KC, Kavazis AN, Deering MA, Falk DJ, Van Gammeren D, Yimlamai T, Ordway GA, Powers SK (2005) Mechanical ventilation induces alterations of the ubiquitin-proteasome pathway in the diaphragm. J Appl Physiol 98:1314-1321

32. Jackman RW, Kandarian SC (2004) The molecular basis of skeletal muscle atrophy. Am J Physiol Cell Physiol 287:C834-C843

33. Di Giovanni S, Molon A, Broccolini A, Melcon G, Mirabella M, Hoffman EP, Servidei S (2004) Constitutive activation of MAPK cascade in acute quadriplegic myopathy. Ann Neurol 55:195-206

34. Latronico N, Peli E, Botteri M (2005) Critical illness myopathy and neuropathy. Curr Opin Crit Care 11:126-132

35. Attaix D, Ventadour S, Codran A, Bechet D, Taillandier D, Combaret L (2005) The ubiquitin-proteasome system and skeletal muscle wasting. Essays Biochem 41:173-186
36. Kandarian SC, Jackman RW (2006) Intracellular signaling during skeletal muscle atrophy. Muscle Nerve 33:155-165

37. Cai D, Frantz JD, Tawa NE Jr, Melendez PA, Oh BC, Lidov HG, Hasselgren PO, Frontera WR, Lee J, Glass DJ, Shoelson SE (2004) IKKbeta/NFkappaB activation causes severe muscle wasting in mice. Cell 119:285-298

38. Fareed MU, Evenson AR, Wei W, Menconi M, Poylin V, Petkova V, Pignol B, Hasselgren PO (2006) Treatment of rats with calpain inhibitors prevents sepsisinduced muscle proteolysis independent of atrogin-1/MAFbx and MuRF1 expression. Am J Physiol Regul Integr Comp Physiol 290:R1589-R1597

39. McDonald MC, Mota-Filipe H, Paul A, Cuzzocrea S, Abdelrahman M, Harwood S, Plevin R, Chatterjee PK, Yaqoob MM, Thiemermann C (2001) Calpain inhibitor I reduces the activation of nuclear factor-kappaB and organ injury/dysfunction in hemorrhagic shock. FASEB J 15:171-186

40. Goll DE, Thompson VF, Li H, Wei W, Cong J (2003) The calpain system. Physiol Rev 83:731-801

41. Machida K, Ishibashi R, Hara T, Ohtsuka A, Hayashi K (2003) Effects of corticosterone on $\mathrm{Ca} 2+$ uptake and myofibrillar disassembly in primary muscle cell culture. Biosci Biotechnol Biochem 67:244-249 